\title{
Obstrucción de la unión ureteropiélica
}

\author{
Ureteropelvic junction (UPJ) obstruction \\ Manuel Atocha Chávez Loya, ${ }^{*}$ Horacio Lozano Zalce, ${ }^{\ddagger}$ Octavio Rodríguez Wyler López ${ }^{\S}$ \\ Citar como: Chávez LMA, Lozano ZH, Rodríguez WLO. Obstrucción de la unión ureteropiélica. \\ Acta Med GA. 2021; 19 (4): 544-546. https://dx.doi.org/10.35366/102543
}

\section{Resumen}

La obstrucción de la unión ureteropiélica (UPJ, por sus siglas en inglés) es la forma más común de obstrucción del tracto urinario en la infancia, puede diagnosticarse de manera prenatal o en la infancia. La hidronefrosis como hallazgo en ultrasonido prenatal es altamente sugestiva del diagnóstico. En infantes los síntomas de infección del tracto urinario son frecuentes, destacan dolor abdominal intermitente en flanco, hematuria y fiebre. Se trata de paciente masculino recién nacido, el ultrasonido prenatal muestra hidronefrosis unilateral, por lo que se practica ultrasonido al nacimiento, observando una marcada pielocaliectasia que termina abruptamente en la unión ureteropiélica, el calibre del uréter distal es normal, hallazgos que prácticamente confirman el diagnóstico en este tipo de pacientes. La ecografía prenatal y postnatal es la herramienta diagnóstica de imagen ideal.

Palabras clave: Obstrucción de la unión ureteropiélica, ureteropielocaliectasia.

\section{INTRODUCCIÓN}

La obstrucción de la unión ureteropiélica (UPJ) es la principal causa de obstrucción del tracto urinario en niños. La hidronefrosis fetal es un hallazgo prenatal altamente sugestivo de obstrucción, el ultrasonido postnatal es la herramienta de imagen de primera línea para hacer el diagnóstico en infantes. El diagnóstico y el tratamiento oportuno permiten limitar el daño, si la función renal no está comprometida el pronóstico es favorable.

\section{Abstract}

Ureteropyelic junction (UPJ) obstruction is the most common form of urinary tract obstruction in children, it can be diagnosed prenatally or in childhood. Hydronephrosis as a prenatal ultrasound finding is highly suggestive for diagnosis. In infants' symptoms of urinary tract infection are frequent, highlighting intermittent abdominal pain in the flank, hematuria, and fever. This is a newborn male patient, the prenatal ultrasound showed hydronephrosis, a postnatal ultrasound is performed that reveals a marked pyelocaliectasis that ends abruptly at the ureteropyelic junction (UPJ) and the caliber of the distal ureter is normal, a finding that practically confirms the diagnosis in this type of patients, therefore prenatal and postnatal ultrasound is the ideal diagnostic imaging tool.

Keywords: Ureteropelvic junction obstruction (UPJ), pelvocaliectasis.
* Residente de Imagenología Diagnóstica y Terapéutica, Facultad de Medicina de la UNAM.

₹ Profesor Titular del Curso de Especialización en Imagenología Diagnóstica y Terapéutica, Facultad de Medicina de la UNAM.

§ Urología Pediátrica.

Hospital Angeles Lomas. México.
Correspondencia:

Manuel Atocha Chávez Loya

Correo electrónico: manuel_atocha9@hotmail.com

Aceptado: 11-11-2020.

www.medigraphic.com/actamedica

\section{CASO CLÍNICO}

Recién nacido (un día) masculino, urología pediátrica solicita ultrasonido renal debido a que en el ultrasonido prenatal se observa hidronefrosis izquierda, único hallazgo descrito. El ultrasonido renal postnatal muestra hidronefrosis grado III unilateral que termina abruptamente en la unión ureteropiélica, el resto del trayecto del uréter no se observa dilatado, ya que presenta un calibre normal; el parénquima renal está intacto, sólo adelgazado (Figura 1). 


\section{DISCUSIÓN}

La obstrucción de la unión ureteropiélica es la forma más común de obstrucción del tracto urinario en niños. Forman parte de las teorías más reconocidas como causas de la obstrucción: la disposición anormal del músculo liso que afecta la distensibilidad del uréter, la inervación anormal del uréter proximal (equivalente de Hirschsprung), cruces vasculares anormales y alguna cicatriz fibrosa en la unión ureteropiélica. Cualquiera puede producir los mismos hallazgos sonográficos característicos de la patología. ${ }^{1,2}$

El diagnóstico puede hacerse prenatal y postnatalmente. El diámetro anteroposterior a nivel de la pelvis renal $>10$ $\mathrm{mm}$ en el feto durante el ultrasonido del tercer trimestre o en el recién nacido sugiere obstrucción. En lactantes y niños con datos de infección del tracto urinario (dolor abdominal intermitente, hematuria y fiebre) el diámetro de la pelvis renal será variable dependiendo del grado de obstrucción, pero de igual forma se observa la pelvis dilatada de manera desproporcionada y los cálices dilatados relativamente uniformes, el parénquima renal no estará afectado, sólo adelgazado. ${ }^{3}$ El ultrasonido es la herramienta diagnóstica inicial y debe practicarse después del periodo de deshidratación fisiológica neonatal, es decir, a las 48 horas de vida postnatal.

El diagnóstico diferencial se hace principalmente con riñón displásico multiquístico, megacálices congénitos, cálculos renales y megauréter. El diagnóstico oportuno es importante, la evolución es incierta y puede mejorar o deteriorarse espontáneamente. ${ }^{4,5}$ Es por ello que la función renal debe ser evaluada luego de seis semanas postnacimiento con un gammagrama renal, usando radiofármacos que se excretan por túbulos renales (MAG 3 o DTPA), facilitando así la estrategia terapéutica.

El tratamiento es amplio, incluye la pieloplastia (cirugía abierta o laparoscópica), el reencaminamiento del cruce vascular, el resecado del segmento estrecho y la aplicación de stents ureterales; por lo que deberá adaptarse a la particularidad del paciente. Es importante saber que después de una cirugía exitosa la pielocaliectasia persiste durante años en la ecografía, esto evitará hacer falsos diagnósticos o menospreciar la mejoría. El éxito quirúrgico en esta etapa del tratamiento debe ser valorado con ayuda de medicina nuclear (gammagrama renal); un tamaño renal en límites normales y el drenaje adecuado garantizan la función renal. La observación del paciente deberá llevarse a cabo en los asintomáticos y con pruebas de función renal normales, ya que ante la presencia de síntomas (dolor, hematuria, fiebre), progresión de la hidronefrosis ultrasonográfica o el deterioro de la función evaluada por gammagrafía deberá ofrecerse un tratamiento oportuno. El pronóstico es excelente si la función renal no está comprometida, puesto que la obstrucción prolongada deteriora la función renal. ${ }^{6-8}$

\section{CONCLUSIÓN}

La obstrucción de la unión ureteropiélica es la principal causa de obstrucción del tracto urinario en la infancia. El ultrasonido prenatal y postnatal es la herramienta de imagen inicial para detectar y caracterizar la dilatación renal. El
Figura 1:

Ultrasonido renal en escala de grises que muestra hidronefrosis grado III, existe dilatación de la pelvis (2.7 $\mathrm{cm}$ ) y de los cálices renales, con una transición abrupta del calibre ureteral en la unión ureteropiélica; el resto del diámetro ureteral se mantiene aparentemente normal. El parénquima renal está adelgazado,

pero permanece intacto, las dimensiones renales están moderadamente agrandadas.
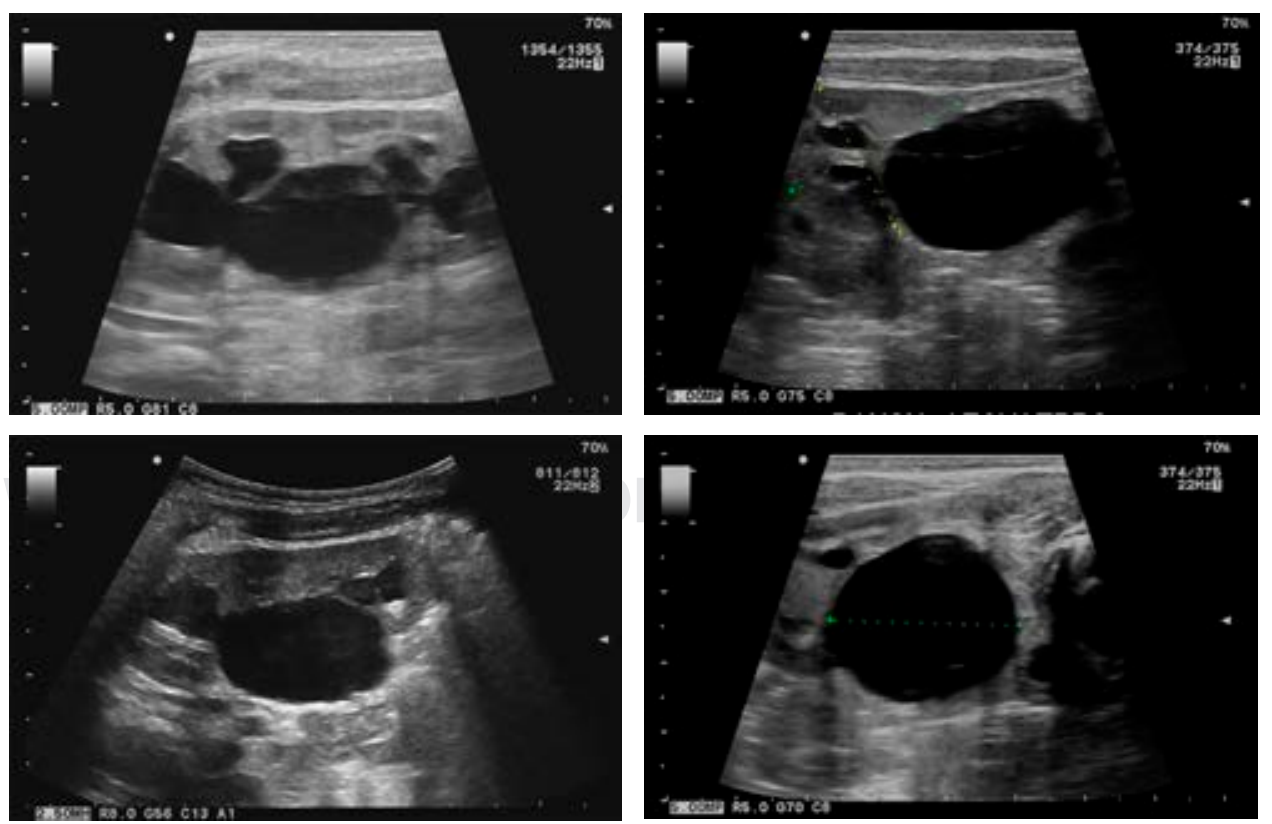
diagnóstico oportuno es importante, pues evitará una evolución incierta y limitará complicaciones. Si el tratamiento garantiza un drenaje renal adecuado, la función renal permanecerá normal, ya que la obstrucción prolongada deteriora la función renal. El pronóstico es excelente si la función renal no está comprometida, la cual será valorada con ayuda de medicina nuclear (gammagrama renal).

\section{REFERENCIAS}

1. Senol C, Onaran M, Gurocak S, Gonul II, Tan MO. Changes in Cajal cell density in ureteropelvic junction obstruction in children. J Pediatr Urol. 2016; 12 (2): 89.e1-5.

2. Rooks VJ, Lebowitz RL. Extrinsic ureteropelvic junction obstruction from a crossing renal vessel: demography and imaging. Pediatr Radiol. 2001; 31 (2): 120-124.

3. Frauscher F, Janetschek G, Helweg G, Strasser H, Bartsch G, zur Nedden D. Crossing vessels at the ureteropelvic junction: detection with contrastenhanced color Doppler imaging. Radiology. 1999; 210 (3): 727-731.
4. Amling CL, O'Hara SM, Wiener JS, Schaeffer CS, King LR. Renal ultrasound changes after pyeloplasty in children with ureteropelvic junction obstruction: long-term outcome in 47 renal units. J Urol. 1996; 156 (6): 2020-2024.

5. Brucher N, Vial J, Baunin C, Labarre D, Meyrignac O, Juricic M et al. Non-contrast-enhanced MR angiography using time-spin labelling inversion pulse technique for detecting crossing renal vessels in children with symptomatic ureteropelvic junction obstruction: comparison with surgical findings. Eur Radiol. 2016; 26 (8): $2697-$ 2704.

6. Li R, Lightfoot M, Alsyouf M, Nicolay L, Baldwin DD, Chamberlin DA. Diagnosis and management of ureteral fibroepithelial polyps in children: a new treatment algorithm. J Pediatr Urol. 2015; 11 (1): 22.e1-6.

7. Mei H, Pu J, Yang C, Zhang H, Zheng L, Tong Q. Laparoscopic versus open pyeloplasty for ureteropelvic junction obstruction in children: a systematic review and meta-analysis. J Endourol. 2011; 25 (5): 727-736.

8. Samarasekera D, Chew BH. Endopyelotomy still has an important role in the management of ureteropelvic junction obstruction. Can Urol Assoc J. 2011; 5 (2): 134-136. 